\title{
TIME SERIES ANALYSIS OF A COMMON HOUSEHOLD ITEM: AN APPLICATION OF BENFORD'S POWER- LAW WITH APPLICATIONS
}

\section{MICHAEL R. GOLINSKI}

2719 Raintree Dr. U10

Fort Collins, Co. 80526

USA

e-mail: zipzip_1999@yahoo.com

\begin{abstract}
Based on cooking times and spatial distributions of common food items in a microwave, I show that the directionality of any food item spinning at a specific rate varies as a function of time. Originally, I assumed that this rate would follow a pattern predicted by Benford's law, in which the orientation of a microwaved item is a function of cooking times (i.e., with higher frequency, low times would lead to a return to the same spatial orientation in the microwave, where higher times would not). In addition, I found that Bendford's law does not apply to non-integer numbers such as fractions (e.g., a cooking time of 1:26 would still use the leading term 1). Therefore, for this example, the cooking time would be 1, and Benford's law would predict that the cooked item would start and stop at the same place (with high probability (high frequency)) on the cooking plate. Alternatively, I hypothesized that given Benford's law, cooking times with higher leading terms would occur less frequently. In what follows, I show that the overall distribution was highly skewed. Given these results, I end the paper by discussing why this pattern may have been observed and how the results of this study could be applied to a wide range of phenomena observed in nature.
\end{abstract}

2010 Mathematics Subject Classification: 37M10.

Keywords and phrases: Benford's law, household item, power-law, spatial orientation, skewed distribution.

Received May 23, 2015

(C) 2015 Scientific Advances Publishers 


\section{Introduction}

A set of numbers is said to satisfy Benford's law (BL) if the leading digit $d(d \in\{1, \ldots, 9\})$ occurs with probability

$$
P(d)=\log _{10}(d+1)-\log _{10}(d)=\log _{10}\left(\frac{d+1}{d}\right)=\log _{10}\left(1+\frac{1}{d}\right) .
$$

Numerically, the leading digits have the following distribution (Figure 1), where $k$ is the leading digit and $P_{-} k$ is the probability $[2,8,9,12,13]$ :

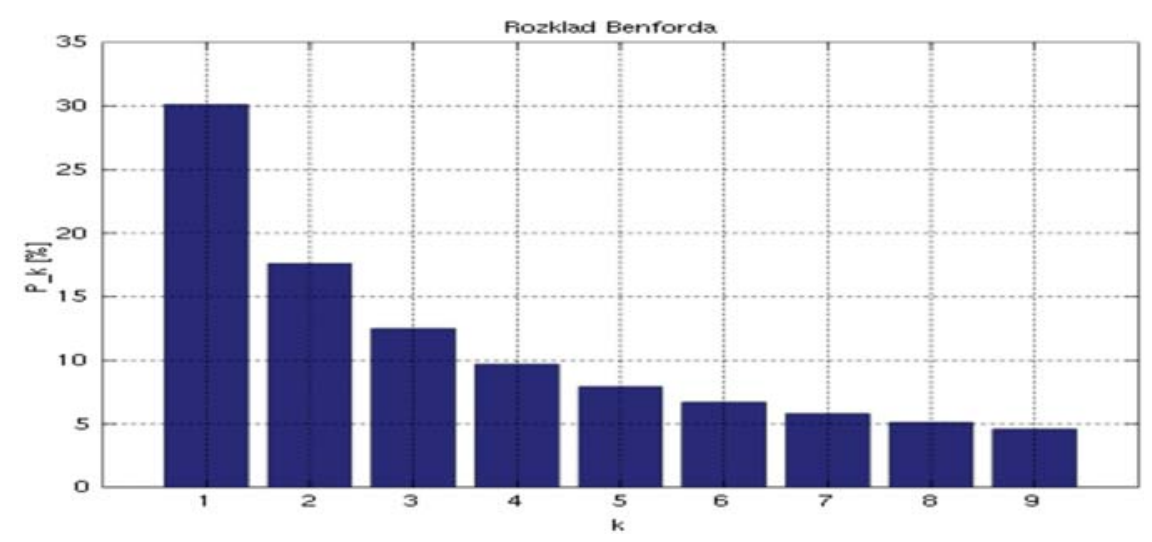

Figure 1. The graph of Benford's law shows that the probability of the leading digit is described by a power law (PL) distribution. One can see that $P$ is high for low digits (or \#s) and decays as a PL as \#s get higher (bounded by 9), which relates to Equation (1).

\section{Materials and Methods}

A Hamilton Beach 1450 Watt microwave was used for the study [15]. The specs of the microwave are as follows: It has an exterior height equal to 17 ", width equal to 14 ". The glass cooking plate has a diameter of 12.4 " and microwave capacity is 0.9 cubic feet, with a depth of 11 " and 10 power levels. The study used only power level 10.

Measurements of time and spatial orientation of microwavable items were made over several days and times of day. A total of $n=45$ items 
were measured for the study. Items measured included $8 \mathrm{oz}$. of coffee in a heat-sealed plastic mug, frozen entrees including $40 \mathrm{oz}$. microwavable macaroni and cheese, lasagna, $8 \mathrm{oz}$. stroganoff and spaghetti marinara all contained in plastic cooking plates. Measurements were also taken of freshly cooked pasta (approximately $16 \mathrm{oz}$.) held in open glass or ceramic bowels and plastic toper were. Equivalents numbers of items of all types mentioned were measured over the duration of the study. Data on the spatial distribution of the microwavable item at the start of cooking time was measured for each item and recorded on an excel spreadsheet. The data was transformed from the measured integer and non-integer values (i.e., cooking times of 1 minute and 36 seconds was transformed to the binary number 1 (the leading term) and cooking times of 2 minutes and 36 seconds was transformed to the number 2 (the leading term) [5]. For long times, e.g., 20 minutes, the leading term was assumed to be 2 . A data column was created to keep track of whether an item started and stopped at the same place in the microwave after a specific cooking time. Thus, the data was either a yes (if the item did return to the same place in the end of microwave cooking time), or a no (if the item did not return to the same place in the end of microwave cooking time). Consequently, the yes and no events were then transformed into real numbers, where 1 was equivalent to a yes event and 0 was equivalent to a no event.

\section{Data Analysis and Results}

Once the data $(n=45)$ was recorded and transformed in excel, there was a column representing the leading term of the cooking time ( $x$-axis), and whether an event did or did not occur $(y$-axis $=$ yes event, $z$-axis $=$ no event) (see Table 1 and Figure 2). 
Table 1. There are four columns, each of which lists variables used in the study. Column 1 lists the real cooking times. Column 2 lists the first digit of the real cooking times. Column 3 lists whether the cooked item started and ended at the same place in the microwave. Column 4 transforms the data in column 3 to binary numbers, where a yes in column 3 (y) corresponds to a 1 in column 4, while a no in column 3 (n) corresponds to a 0 in column 4

\begin{tabular}{|c|c|c|c|}
\hline Time & $\begin{array}{l}\text { First time } \\
\text { digit }\end{array}$ & $\begin{array}{l}\text { Start and end of process at same place in } \\
\text { microwave }\end{array}$ & $\begin{array}{l}\text { no }=0, \text { yes } \\
=1\end{array}$ \\
\hline $0: 45$ & 4 & 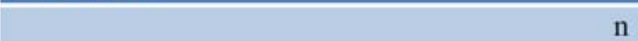 & 0 \\
\hline $1: 20$ & 1 & $\mathrm{y}$ & 1 \\
\hline $1: 10$ & 1 & $\mathrm{n}$ & 0 \\
\hline $1: 20$ & 1 & $\mathrm{y}$ & 1 \\
\hline $2: 00$ & 2 & $\mathrm{y}$ & 1 \\
\hline $1: 14$ & 1 & $\mathrm{n}$ & 0 \\
\hline $1: 13$ & 1 & $\mathrm{n}$ & 0 \\
\hline$: 30$ & 3 & $\mathrm{y}$ & 1 \\
\hline$: 25$ & 2 & $\mathrm{n}$ & 0 \\
\hline $1: 00$ & 1 & $\mathrm{y}$ & 1 \\
\hline $1: 00$ & 1 & $\mathrm{y}$ & 1 \\
\hline$: 20$ & 2 & $\mathrm{y}$ & 1 \\
\hline$: 25$ & 2 & $\mathrm{n}$ & 0 \\
\hline$: 25$ & 2 & $\mathrm{n}$ & 0 \\
\hline $1: 11$ & 1 & $\mathrm{y}$ & 1 \\
\hline$: 41$ & 4 & $\mathrm{y}$ & 1 \\
\hline $1: 30$ & 1 & $\mathrm{y}$ & 1 \\
\hline $1: 23$ & 1 & $\mathrm{n}$ & 0 \\
\hline $1: 21$ & 1 & $y$ & 1 \\
\hline $1: 26$ & 1 & $\mathrm{n}$ & 0 \\
\hline$: 25$ & 2 & $\mathrm{n}$ & 0 \\
\hline $1: 20$ & 1 & $y$ & 1 \\
\hline$: 38$ & 3 & $\mathrm{y}$ & 1 \\
\hline $1: 24$ & 1 & $\mathrm{n}$ & 0 \\
\hline $1: 30$ & 1 & $y$ & 1 \\
\hline $1: 21$ & 1 & $\mathrm{y}$ & 1 \\
\hline $1: 19$ & 1 & $\mathrm{n}$ & 0 \\
\hline $1: 22$ & 1 & $\mathrm{n}$ & 0 \\
\hline $1: 13$ & 1 & $\mathrm{n}$ & 0 \\
\hline $1: 18$ & 1 & $\mathrm{n}$ & 0 \\
\hline $1: 20$ & 1 & $\mathrm{y}$ & 1 \\
\hline $16: 00$ & 1 & $\mathrm{y}$ & 1 \\
\hline$: 47$ & 4 & $\mathrm{n}$ & 0 \\
\hline $1: 17$ & 1 & $\mathrm{n}$ & 0 \\
\hline $1: 18$ & 1 & $\mathrm{n}$ & 0 \\
\hline $1: 20$ & 1 & $\mathrm{y}$ & 1 \\
\hline $1: 17$ & 1 & $\mathrm{n}$ & 0 \\
\hline $1: 18$ & 1 & $\mathrm{n}$ & 0 \\
\hline
\end{tabular}


Table 1. (Continued)

\begin{tabular}{|c|c|c|c|}
\hline $1: 20$ & 1 & $y$ & 1 \\
\hline $1: 17$ & 1 & $\mathrm{n}$ & 0 \\
\hline $1: 18$ & 1 & $\mathrm{n}$ & 0 \\
\hline $1: 40$ & 1 & $y$ & 1 \\
\hline$: 33$ & 3 & $\mathrm{n}$ & 0 \\
\hline $5: 00$ & 5 & $y$ & 1 \\
\hline $6: 00$ & 6 & $y$ & 1 \\
\hline $7: 00$ & 7 & $y$ & 1 \\
\hline $8: 00$ & 8 & $\mathrm{y}$ & 1 \\
\hline $9: 00$ & 9 & $\mathrm{y}$ & 1 \\
\hline
\end{tabular}

Frequency of a yes (1) or no (0) event was calculated in Microsoft Excel by using the following equation:

$$
f=\frac{1}{\sum \text { all events }(0 \text { or } 1)} .
$$

Equation (2) [16] was then treated as the independent variable ( $y$-axis) and plotted against the dependent variable ( $x$-axis), i.e., the number of times the real numbers $(1, \ldots, 9)$, which represent the leading term of the cooking time of each item in the microwave occurred with frequency $f$ $[1,16]$. Figure 2 shows a skewed pattern not indicative of power law behaviour. However, the skewness that occurs as the first digit gets larger, may be indicative of power law like behaviour. Future analysis is needed. 


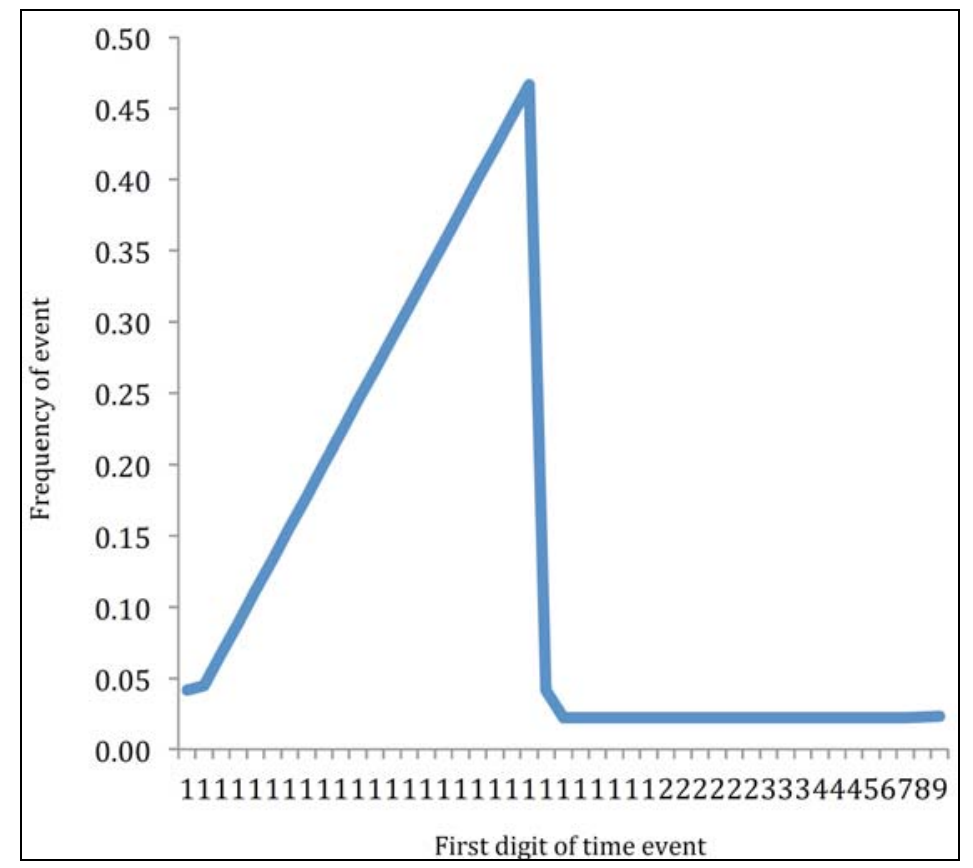

Figure 2. Plot of first digit of time event (cooking time in microwave) vs. frequency of event, where the event is the frequency at which the food or beverage item remained in the same spatial orientation within the microwave at the start and end of the cooking event.

\section{Discussion and Conclusions}

There may be several reasons why my initial hypothesis was falsified, in that low first digit numbers showed no higher probability of occurring than high first digit numbers [7]. (1) The plate rotation within the microwave may have been too variable, which resulted in the fact that the BL pattern was not observed. (2) The types of food and beverages, including their weights (grams vs. volumetric) may have added too much variation, resulting in the deviation from the observation of BL patterns. (3) Certain mechanical properties of the microwave itself, including its power level, exterior height, width, capacity, exterior depth, and diameter of the cooking plate could have all affected the observed data [15]. 
It is also quite possible that I did not record enough data for higher number events [3]. Much of the data consists of cooking times for coffee, tea, and food items that take no more than five minutes to cook. Hence, this could weight the data in such a way as to draw conclusions based on a small sample size of higher events (see Figure 2). In addition, I used data consisting of fractional (non-integer) cooking times (e.g., 33 minutes), which corresponds to negative log numbers, where all cooking time with leading terms in integer form (e.g., 1:33 minutes) have zero or positive $\operatorname{logs}$ (e.g., $\log 1$ equals 0). Obviously, this may conflict with the assumptions of Equation (1) [6]. Clearly, further research is needed.

Although my study may seem trivial at first, I believe it is the simplest of experiments that can shed light on higher order studies, and may hold a great deal of power for describing many patterns generated by more complex phenomena observed in nature [10, 14]. Given the caveats discussed above, it should be noted that the BL pattern has been observed in many naturally occurring systems, not limited to: outcomes of exponential growth processes, such as the exponential growth of bacteria [8, 10]. (1) For example, an exponentially growing quantity is moving towards the right side on a log-scale at a constant rate. If one where to measure the number of bacteria at a random time in, for example, a 10-day window, one might get a random point on the logscale, uniformly distributed in that corresponding window. Hence, one would expect this kind of probability distribution to satisfy BL, and the classic power-law behaviour would be observed. (2) Another example would be the existence of the BL pattern used to describe real-world distributions that span several orders of magnitude rather uniformly, e.g., population densities of cities, and the spread of disease through these populations [11], both of which are likely to satisfy the power-law pattern described by BL [11].

Clearly, there are many more examples from naturally occurring systems that could be discussed. However, for the sake of brevity, I will end my discussion here. 


\section{References}

[1] Steven W. Smith, The Scientist and Engineer's Guide to Digital Signal Processing, Chapter 34, Explaining Benford's Law, Retrieved 15 December 2012.

[2] R. M. Fewster, A simple explanation of Benford's law, The American Statistician 63(1) (2009), 26-32.

[3] Paul H. Kvam and Brani Vidakovic, Nonparametric Statistics with Applications to Science and Engineering, p. 158.

[4] Frank Benford, The law of anomalous numbers, Proceedings of the American Philosophical Society 78(4) (1938), 551-572.

[5] Simon Newcomb, Note on the frequency of use of the different digits in natural numbers, American Journal of Mathematics 4(1/4) (1881), 39-40.

[6] Theodore P. Hill, A statistical derivation of the significant-digit law (PDF), Statistical Science 10 (1995), 354-363.

[7] Arno Berger and Theodore P Hill, Benford's Law Strikes Back: No Simple Explanation in Sight for Mathematical Gem, 2011.

[8] Theodore P. Hill, The first digit phenomenon, (PDF), American Scientist 86(4) (1998), 358.

[9] Roger S. Pinkham, On the distribution of first significant digits, Ann. Math. Statist. 32(4) (1961), 1223-1230.

[10] A. K. Formann and Richard James Morris, ed., The Newcomb-Benford law in its relation to some common distributions, PLoS ONE 5(5) (2010).

[11] L. M. Leemis, B. W. Schmeiser and D. L. Evans, Survival distributions satisfying Benford's law, The American Statistician 54(4) (2000), 236-241.

[12] L. Dümbgen and C. Leuenberger, Explicit bounds for the approximation error in Benford's law, Elect. Comm. in Probab. 13 (2008), 99-112.

[13] R. A. Raimi, The first digit problem, American Mathematical Monthly 83 (1976), 521-538.

[14] C. Durtschi, W. Hillison and C. Pacini, The effective use of Benford's law to assist in detecting fraud in accounting data, J. Forensic Accounting 5 (2004), 17-34.

[15] David M. Pozar, Microwave Engineering, Addison-Wesley Publishing Company, 1993.

[16] D. C. Giancoli, Physics for Scientists and Engineers, 2nd Edition, Prentice Hall, 1988. 